\title{
Edema agudo de pulmón de reentrada a gran altitud
}

\author{
José Antonio Viruez-Soto* 1,a; Amílcar Tinoco-Solórzano 2,3,4,a
}

RESUMEN

El edema agudo de pulmón de altura es una condición médica potencialmente mortal. Se conoce también como edema agudo de pulmón de altura "de reentrada" y puede ocurrir en residentes de la altura que retornan de una estadía en una zona de menor altitud. Presentamos el caso de un paciente de sexo masculino de 14 años de edad, nativo y residente de la ciudad de El Alto (La Paz, Bolivia), a 4090 metros sobre el nivel del mar, que presenta un cuadro de edema agudo pulmonar de altura a su regreso de una localidad a 500 metros sobre el nivel del mar. Este paciente requirió tratamiento intensivo y tuvo una evolución favorable.

Palabras clave: Edema pulmonar; Altitud; Población residente (Fuente: DeCS BIREME).

\section{Acute re-entry high-altitude pulmonary edema}

\section{ABSTRACT}

Acute high-altitude pulmonary edema is a potentially life-threatening medical condition. Also known as acute re-entry high-altitude pulmonary edema, it may occur in highland dwellers who return to their hometown after having stayed at a lower-level area. This is the case of a 14 year-old male patient, native to and resident of the city of El Alto (La Paz, Bolivia), located at 4,090 meters above sea level. The patient developed acute high-altitude pulmonary edema when returning from a town at 500 meters above sea level. He required intensive care and showed a favorable evolution.

Keywords: Pulmonary edema; Altitude; Resident population (Source: MeSH NLM).

\footnotetext{
1 Hospital del Norte El Alto, Departamento de Apoyo Crítico. La Paz, Bolivia.

2 EsSalud, Hospital Nacional Ramiro Prialé Prialé, Servicio de Cuidados Intensivos e Intermedios. Huancayo, Perú.

3 Universidad de San Martin de Porres, Facultad de Medicina Humana, Centro de Investigación de Medicina de Altura. Huancayo, Perú.

4 Universidad Peruana Los Andes, Facultad de Medicina Humana, Cátedra de Medicina Interna II. Huancayo, Perú.

a Médico especialista en Medicina Intensiva.

* Autor corresponsal
} 


\section{INTRODUCCIÓN}

Las regiones de gran altura son el hogar de alrededor de 300 millones de personas en el mundo ${ }^{(1,2)}$. La exposición a un ambiente con baja presión de oxígeno origina una serie de reacciones fisiológicas respiratorias y cardiovasculares, así como el uso del oxígeno a través de un complejo proceso de aclimatación (3-5). El edema agudo de pulmón de altura (EAPA) es una condición médica potencialmente mortal, su incidencia es de un habitante por cada 10000 pobladores y ocasiona la mayor parte de muertes relacionadas a las enfermedades de gran altura ${ }^{(6)}$. El EAPA es un tipo de edema no cardiogénico que ocurre casi siempre de 2 a 4 días después del ascenso por encima de los 2500-3000 metros y rara vez aparece después de 4 o 5 días a la misma altitud debido a la aclimatación que se produce ${ }^{(1,7)}$.

El EAPA se presenta en dos formas, la primera, en habitantes "no aclimatados previamente" de tierras bajas que ascienden rápidamente; y la segunda, en residentes de la altura que retornan de un nivel más bajo de altura, y que también es conocida como edema agudo de pulmón de altura "de reentrada". La incidencia de esta última llega casi al 0,6\% en adultos, con predominio de varones jóvenes $(84 \%)^{(2,7-9)}$. El mecanismo de este cuadro es el de una vasoconstricción pulmonar hipóxica heterogénea que expone a los capilares pulmonares a alta presión con daño de sus paredes, lo cual lleva a una fuga capilar y edema por alta permeabilidad con presencia de proteínas y eritrocitos en los alvéolos ${ }^{(2,10,11)}$.

En cuanto al tratamiento, uno de los regímenes de prevención incluye el uso de nifedipino como bloqueante cálcico e inhibidor de vasoconstricción pulmonar hipóxica, el cual produce vasodilatación pulmonar que detiene el aumento de la presión arterial pulmonar. El sildenafil, que reduce la incidencia de vasoconstricción pulmonar hipóxica, es también eficaz en la prevención y el tratamiento. La dexametasona también disminuye significativamente la elevación de la presión arterial pulmonar a grandes alturas ${ }^{(1,2,12)}$.

\section{CASO CLÍNICO}

Paciente masculino de 14 años, estudiante de secundaria, procedente y residente de la ciudad de El Alto, La Paz (Bolivia) a 4090 metros sobre el nivel del mar (m s. n. m.), sin antecedentes médicos de importancia. Realiza un viaje a la localidad de Palos Blancos (500 m s. n. m.) durante 15 días en las vacaciones de invierno. Al retornar por vía terrestre presenta un cuadro clínico de 2 horas de evolución caracterizado por malestar general, palidez, náuseas que llegan al vómito, tos productiva con expectoración mucosa que se torna rojiza (asalmonada) por lo que es admitido en Urgencias y, posteriormente, en la Unidad de Terapia Intensiva.

Al ingreso, el paciente está en muy mal estado general, somnoliento y desorientado en espacio y tiempo, importante dificultad respiratoria con uso de musculatura accesoria, taquipnea de 55 ciclos por minuto, saturación periférica de $30 \%$ sin aporte de oxígeno y que llega a 70 \% con aporte de oxígeno por mascarilla con bolsa reservorio, ruidos respiratorios disminuidos con estertores húmedos de pequeña burbuja. Se procede a intubación orotraqueal y se extrae abundante cantidad de material asalmonado espumoso, luego se conecta a un ventilador mecánico y se inicia ventilación protectora pulmonar.

La gasometría de ingreso muestra $\mathrm{pH}=7,39 ; \mathrm{pO}_{2}=34$, $\mathrm{pCO}_{2}=19, \mathrm{HCO}_{3}=18$, Sat $55 \%$ e índice PaFi de 68. Durante la hospitalización, el paciente recibe dexametasona $8 \mathrm{mg}$ IV c/12 h, acetazolamida $250 \mathrm{mg}$ por SNG c/12 h, sildenafil $50 \mathrm{mg}$ por SNG c/12 h y vitamina C $1 \mathrm{~g}$ IV c/día. Permanece en Unidad de Terapia Intensiva durante $48 \mathrm{~h}$. El control gasométrico muestra $\mathrm{pH}=7,36 ; \mathrm{pO}_{2}=142, \mathrm{pCO}_{2}=23$, $\mathrm{HCO}_{3}=19,2$; Sat 98,5\%, índice $\mathrm{PaFi}=355$.

Fue desconectado y extubado del ventilador mecánico con éxito a las 72 horas para llevarlo a la sala común. La ecocardiografía transtorácica descarta alteraciones fisiológicas y anatómicas cardiacas. Esta prueba se realizó $48 \mathrm{~h}$ después del ingreso, porque en el momento de la hospitalización el equipo que realiza la prueba no estaba disponible.

El paciente fue dado de alta en buen estado general 48 horas después. La comparativa de las radiografías de tórax de la evolución se encuentran en la figura 1: en la radiografía (A) se evidencia una evidente congestión pulmonar hiliar con opacificaciones bilaterales diseminadas y una prominencia importante de arteria pulmonar indicativa de hipertensión pulmonar. Todos los hallazgos anteriores son compatibles con edema pulmonar, en contraste con la segunda radiografía (B) que muestra los pulmones mejor aireados sin las alteraciones previamente descritas. Así también, el electrocardiograma de ingreso evidencia el desvío del eje hacia la derecha $\left(+150^{\circ}\right)$ y la inversión de la onda $\mathrm{T}$ en precordiales $\mathrm{V} 1$ y V2 que son representativos de sobrecarga derecha aguda (Figura 2). 


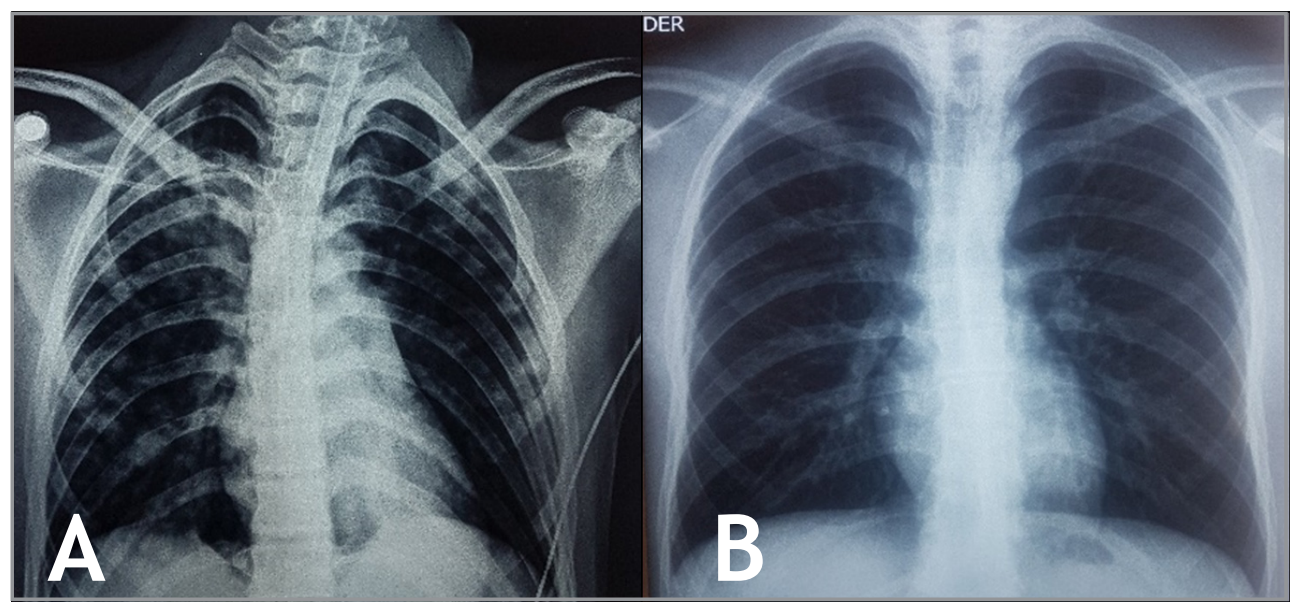

Figura1. Radiografía de tórax al ingreso (A) y al egreso (B) del paciente de la Unidad de Terapia Intensiva (adultos)

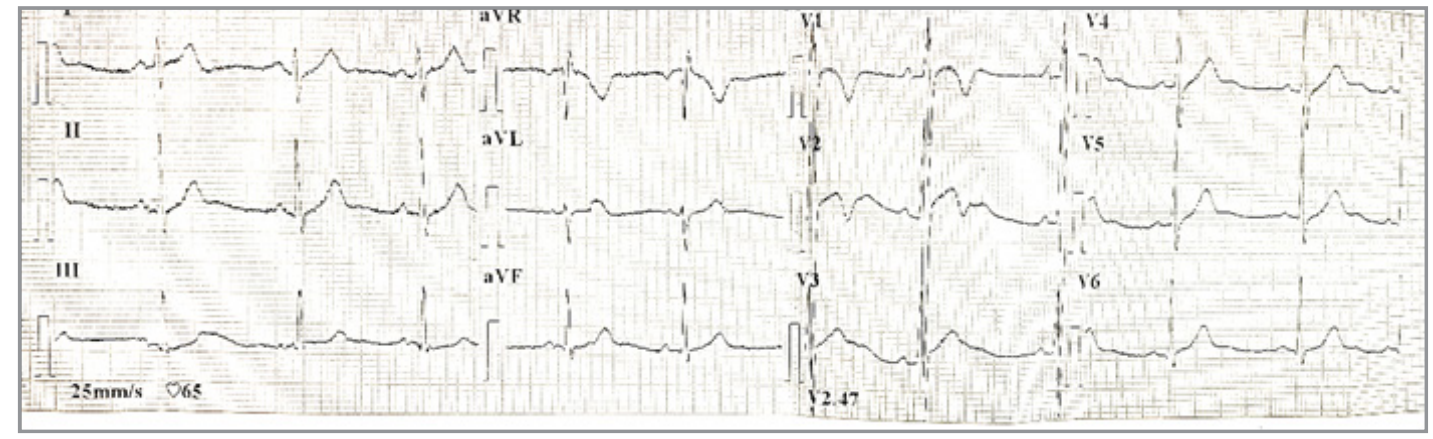

Figura 2. Electrocardiograma del paciente

\section{DISCUSIÓN}

Este trabajo ofrece interesantes datos acerca de un caso de edema agudo de pulmón "de reentrada" a gran altura; como la rapidez de la instauración del cuadro, prácticamente durante el viaje de regreso al lugar de residencia, y no a los 2 o 3 días posteriores al retorno, como se reporta clásicamente. De forma característica, el cuadro se presentó en un joven residente en una gran altura (en concordancia con la literatura), quien permaneció por un periodo de 2-3 semanas a una altitud menor. Si bien la acetazolamida es un fármaco útil en la prevención del edema agudo de pulmón de altura, en el presente caso se asoció a una evolución favorable por probable reversión de la vasoconstricción pulmonar hipóxica que se presenta en estos pacientes, por lo cual debería considerarse como parte del tratamiento de primera elección ${ }^{(13,14)}$. Por otro lado, existen reportes recientes acerca de la utilidad de antioxidantes (como la vitamina C) que también disminuyen la incidencia de vasoconstricción pulmonar hipóxica, el paciente recibió este fármaco durante su atención (15). El hecho de que las personas afectadas por este cuadro requieran permanecer por lo menos 2 semanas en alturas menores sugiere una pérdida o alteración en los procesos "aclimatación" como causantes del cuadro (16). Se destaca también que no se administró nifedipino ya que se relaciona a la prevención del cuadro, principalmente, pero no se emplean en el tratamiento. El manejo correcto, tras el reconocimiento oportuno, con base en la evidencia permitió devolver al paciente a su entorno social en condiciones fisiológicas adecuadas.

Contribuciones de los autores: José Antonio Viruez Soto, y Amilcar Tinoco Solórzano participaron en la generación y diseño del estudio, recolección, análisis e interpretación de los datos así como la redacción del artículo.

Fuentes de financiamiento: Este artículo ha sido financiado por los autores.

Conflicto de interés: Los autores declaran no tener ningún conflicto de interés.

\section{REFERENCIAS BIBLIOGRÁFICAS}

1. Bhagi S, Srivastava S, Singh SB. High-altitude pulmonary edema: review. J Occup Health. 2014; 56(4): 235-43.

2. Jones BE, Stokes S, McKenzie S, Nilles E, Stoddard GJ. Management 
of high altitude pulmonary edema in the Himalaya: a review of 56 cases presenting at pheriche medical aid post $(4240 \mathrm{~m})$. Wilderness Environ Med. 2013; 24(1): 32-6.

3. Fulco CS, Beidleman BA, Muza SR. Effectiveness of preacclimatization strategies for high-altitude exposure. Exerc Sport Sci Rev. 2012; 41(1): 55-63.

4. Srivastava S, Dwivedi S. Significance of renin angiotensin aldosterone system (RAAS) pathway in high altitude pulmonary edema (HAPE) susceptibility. J Clin Mol Endocrinol. 2016; 1(3): 1-4.

5. Netzer N, Strohl K, Faulhaber M, Gatterer H, Burtscher M. Hypoxiarelated altitude illnesses. J Travel Med. 2013; 20(4): 247-55.

6. Gupta RK, Himashree G, Singh K, Soree P, Desiraju K, Agrawal A, et al. Elevated pulmonary artery pressure and brain natriuretic peptide in high altitude pulmonary edema susceptible nonmountaineers. Sci Rep. 2016; 6: 21357.

7. Bhattachar SA, Singhal S, Paliwal V, Malhotra VK, Kotwal A. High altitude pulmonary edema (HAPE) with pulmonary embolism. Hearth India. 2016; 4: 23-5.

8. Luks AM, Swenson ER, Bärtsh P. Acute high-altitude sickness. Eur Respir Rev. 2017; 26(143).

9. Pandey P, Lohani B, Murphy H. Pulmonary embolism masquerading as high altitude pulmonary edema at high altitude. High Alt Med Biol. 2016; 17(4): 353-8.

10. Dehnert C, Mereles D, Greiner S, Albers D, Scheurlen F, Zügel S, et al. Exaggerated hypoxic pulmonary vasoconstriction without susceptibility to high altitude pulmonary edema. High Alt Med Biol. 2015; 16(1): 11-7.

11. Lorenzo FR, Huff C, Myllymäki M, Olenchock B, Swierczek S, Tashi $\mathrm{T}$, et al. A genetic mechanism for tibetan high-altitude adaptation. Nat Genet. 2014; 46(9): 951-6.

12. Bärtsch P, Swenson ER. Clinical practice: acute high-altitude illnesses. N Engl J Med. 2013; 368(24): 2294-302.

13. Luks AM, McIntosh SE, Grissom CK, Auerbach PS, Rodway GW, Schoene RB, et al. Wilderness medical society practice guidelines for the prevention and treatment of acute altitude illness: 2014 update. Wilderness Environ Med. 2014; 25 (Suppl. 4): S4-14.

14. Spielvogel H. Edema agudo pulmonar y de altura. Cuadernos. 2006; 51(2): 73-9.

15. Cerny D, Rexhaj E, Bouillet E, Soria R, Meister T, Villena M, et al. Sustained hypoxic pulmonary hypertension and systemic vascular dysfunction in high-altitude dwellers prone to re-entry high altitude pulmonary edema, improvement by anti-oxidants. FASEB J. 2015; 29(1): 1051-6.
16. Zafren K. Prevention of high altitude illness. Travel Med Infect Dis. 2014; 12(1): 29-39.

\section{Correspondencia:}

José Antonio Viruez Soto

Dirección: Avenida Juan Pablo II Número 220, Zona Rio

Seco, El Alto. La Paz, Bolivia.

Correo electrónico: antonioviruez@hotmail.com

Teléfono: +53178804139

Recibido: 21 de junio de 2019

Evaluado: 11 de julio de 2019

Aprobado: 26 de noviembre de 2019

(c) La revista. Publicado por Universidad de San Martín de Porres, Perú.

(c) $\mathbf{\text { Br }}$ Licencia de Creative Commons Artículo en acceso abierto bajo términos de Licencia Creative Commons Atribución 4.0 Internacional. (http://creativecommons.org/licenses/by/4.0/)

\section{ORCID iDs}

José Antonio Viruez Soto

(1) https: / / orcid.org/0000-0002-0579-5186

Amilcar Tinoco Solórzano

(ㄱ) https: / / orcid.org/0000-0002-4609-3455 